\title{
Long Term Reproducibility Check of a Full Field Digital
}

\section{Mammography System}

\author{
Francesca Pietrobon ${ }^{1}$, Salvatore Panarello ${ }^{2}$, Orazio De Toffol ${ }^{2}$, Marco Toigo $^{2}$ and Elena Bellan ${ }^{3}$ \\ 1. UOS Department of Medical Physics, S. Martino Hospital, Belluno 32100, Italy \\ 2. UOC Department of Diagnostic Radiology, S. Maria del Prato Hospital, Feltre 32032, Italy \\ 3. UOSD Department of Medical Physics, S. Maria Misericordia Hospital, Rovigo 45100, Italy
}

\begin{abstract}
In this study different types of customized phantoms were used to examine a long term reproducibility check of a full field digital mammography system. These phantoms can be distinguished into three types: a PMMA (polimethylmethacrylate) object for an easy signal-to-noise ratio measurement in a defined region, a phantom with particular details of various dimensions (TORMAS) and a phantom for a contrast-detail analysis (CDMAM). In our center the ARTINIS ANALYZER software has been used since 2011 to weekly perform a CDMAM phantom analysis. This software is based on the original CDCOM algorithm suggested by the EUREF, the European Reference Organization for assured quality breast screening and diagnostic services. However the CDMAM phantom is a contrast-detail object designed for image quality type tests and not for long term reproducibility control. Since 2013, as suggested by the guidelines of Veneto region, a TORMAS weekly acquisition and analysis of data has also been performed. The purpose of this study is to discuss the data obtained by using the two different detail phantoms, CDMAM and TORMAS. These results can provide some correlations among the analyzed parameters comparing them with the ones emerging from the PMMA phantom analysis. The study reveals that the basic PMMA phantom is the object that provides the necessary information about the stability of the exposure parameters and the reproducibility of a mammographic equipment in terms of image quality indexes.
\end{abstract}

Key words: Contrast-detail phantoms, digital mammography, long-term reproducibility.

\section{Introduction}

Long term reproducibility check consists in simple tests. They could be implemented in a digital radiological equipment to monitor the stability of the system performances based on the exposure parameters and the related digital image quality. To check the repeatability of the results the single periodic test is performed under identical conditions of radiological exposure. Initially, analyzing a group of images, a set of certain parameters is defined as the baseline data and is compared to the next images.

In a periodic measurement the identified image parameters are matched with the same types of indexes from the baseline data. If the comparison between the two values of a parameter is into a

Corresponding author: Francesca Pietrobon, medical physicist, research fields: radiology, radiotherapy, and radioprotection. tolerance range, the system can be considered stable. Otherwise some corrective procedures have to be applied.

Several phantoms have been suggested for long term reproducibility control of mammographic images. Some of these phantoms [1-3] are suitable for screen-film systems and have been adapted to modern digital detectors. Others are designed for digital systems and for dedicated applications like the tomosynthesis [4].

For long term reproducibility check the test has to be simple and consistent. The results of the test in terms of quality of the indexes, calculated based on the digital images produced by the detector, are directly correlated to the exposure parameters to detect possible anomalies.

For the digital mammographic systems the producer generally suggests the same basic procedure even 
recommended by the European guidelines [5]. This procedure involves the exposure of a typical 40-45 mm PMMA (polymethylmethacrylate) and the measurement of the MPV (mean pixel value), the SD (standard deviation) and the signal-to-noise-ratio. These data are collected for a defined ROI (region-of-interest) with an area of about $1 \mathrm{~cm}$ (square). The ROI is placed in the middle of the digital image at about $6 \mathrm{~cm}$ from the chest wall side. This procedure allows to monitor the acquisition system stability in terms of exposure parameters and to correlate them with the repeatability of the SNR values.

In the "Quality Assurance Protocol for mammographic Screening" applied to digital mammographic equipments in the Italian region of Veneto [6] the long term reproducibility control is checked through the use of the TORMAS phantom [7]. The parameters are weekly analyzed via web by the AUTOPIA software [8]. The exposure conditions to image the phantom are defined by considering the type of the equipment that has to be controlled. The purpose is to mimic the results obtainable in a clinical situation by using phantoms with the same thickness of the breast but also with high density details. As a consequence, considering the total equipments scenario, some exposures are performed through a completely automatic mode, other exposures use a semiautomatic mode and others a manual mode [6, 14].

Besides the TORMAS acquisition as suggested by Veneto's guidelines, in our center a CDMAM ver. 3.4 phantom $[9,10]$ independent analysis has been automatically performed. The data had been weekly collected by the dedicated software ARTINIS [10] based on the original CDCOM analysis, which was suggested by the EUREF. However the CDMAM phantom is a contrast detail object designed for image quality type tests of a mammograhic equipment and not for long term reproducibility control.

The purpose of this study is to discuss the data obtained by using the two different detail phantoms, CDMAM and TORMAS, comparing against PMMA data. These results can provide some correlations among the analyzed parameters.

The method involves three phases:

(1) The analysis of long term reproducibility of the threshold contrast visibility. This first analysis is performed using the standard number of CDMAM images suggested in the beginning part of automated scoring to simulate human readout (first phase of image analysis).

(2) The comparison between the data of CDMAM and TORMAS phantoms based on the reproducibility of image quality parameters (second phase of image analysis).

(3) The evaluation of the reproducibility of the threshold contrast automated measurement for the CDMAM phantom. This procedure is carried out using a larger number of images than during the first phase (third phase of image analysis).

All DICOM images have been analyzed in raw format. The correct type of images to be analyzed is specified by the attribute "For Processing" in the relative DICOM header tag.

The whole statistical analysis has been performed using Statistica 6.1 software package.

\section{Materials and Methods}

\subsection{CDMAM phantom and ARTINIS Software}

The CDMAM phantom ver. 3.4 is a contrast-detail phantom with cylindrical gold objects simulating clinical microcalcifications, arranged in 205 cells organized in 16 gold thickness with a range $0.03-2 \mathrm{~m}$ and 16 object diameter with a range 0.06-2 $\mathrm{mm}$. Each cell has a disk at the center of the area and another one randomly positioned at one corner of the little square. The total thickness of CDMAM, equipped with its total PMMA layers, simulates $50 \mathrm{~mm}$ of PMMA [10, 12].

When the phantom was commercially introduced, the images were analyzed by human observers in 
multiple readings and the result was averaged over the observers by applying the NNC procedure (nearest neighbor correction) in the 205 cells $[11,12]$. However, the human readout has many disadvantages like: time-consuming reading, inter-reader variability, learning effects to know the correct position of the disk in the right corner, and the NCC correction does not seem to be the best scheme to obtain contrast-detail curves.

An automatic reading software (ARTINIS Analyzer) is now supplied with the phantom $[10,12]$ : it is possible to upload a number of CDMAM images, acquired by applying little shifts of the phantom along the bucky, to obtain directly an average reading of the 205 cells.

The automatic reading mimics an observer analysis through a particular psychometric interpolation applied in the range of diameter 0.1-1 $\mathrm{mm}$ [12]:

$$
p(d)=\frac{0.75}{1+e^{f\left(C-C_{T}\right)}}+0.25
$$

where, $p(d)$ is the probability of detection as a function of diameter $d, C$ is the signal contrast $C=$ $\log \left(1-e^{-\mu d}\right)$ with $\mu=0.190 \mu \mathrm{m}^{-1}$ that is the linear coefficient of gold, $C_{T}$ is the contrast threshold corresponding to $p=0.625$, $\mathrm{f}$ is a fitted parameter.

In the Supplement number 1 of "European Guidelines for quality assurance in breast cancer screening and diagnosis", titled [13] "European Protocol for the quality control of the physical and technical aspects of mammographic screening", it is suggested to acquire at least 16 CDMAM images for every control by moving the phantom over the bucky and setting a detection rate of $62.5 \%$, that is the mean value between the random probability to match the circular golden object in the corner of a cell $(25 \%)$ and the perfect probability to match the same object in the correct corner of a cell (100\%).

The results of phantom image analysis are a contrast-detail curve and an "image quality index" IQ proportional to the reciprocal of the area under the contrast-detail curve. In particular, the threshold gold thickness in the image quality measurements for the $0.1 \mathrm{~mm}$ diameter disk [13] corresponds to the limiting values for SDNR (signal-difference-to noise ratio) and it has been employed as a quantitative parameter in the further analysis (named simply “contrast@0 0.1 mm CDMAM" in the following text).

\subsection{PMMA Phantom}

The SNR (signal-to-noise-ratio) is an objective and simple measurement of the mean pixel value divided by the standard deviation evaluated in a reference region of interest with an area of about $1 \mathrm{~cm}^{2}$ placed at about $6 \mathrm{~cm}$ from the chest wall. This evaluation is, usually, performed on digital images of a PMMA phantom with a typical thickness of 40-45 $\mathrm{mm}$. In this work we have used a $40 \mathrm{~mm}$ PMMA thick.

\subsection{TORMAS Phantom and AUTOPIA Software}

TORMAS phantom (Fig. 1) is a series of PMMA

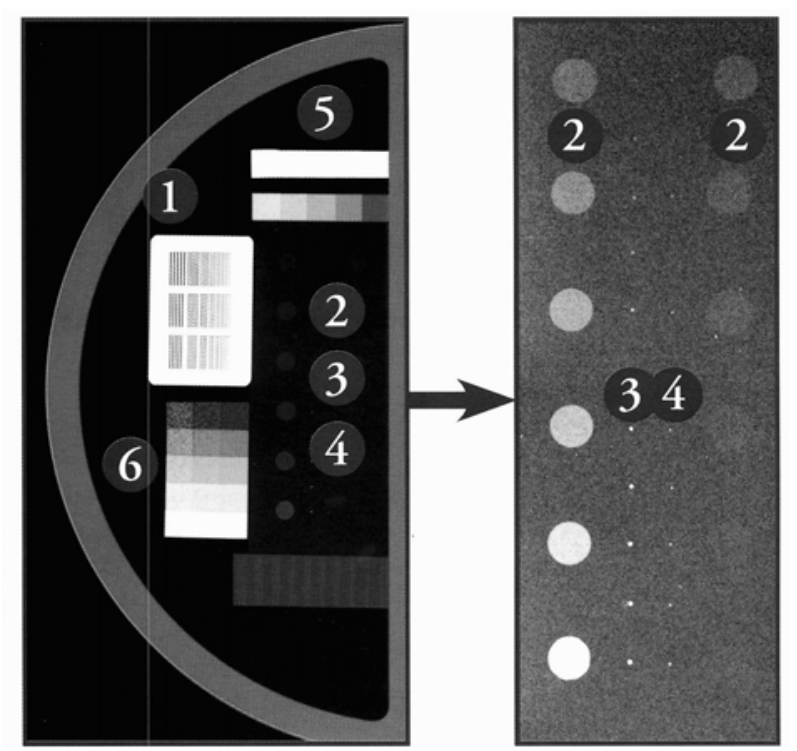

Fig. 1 Scheme of the TORMAS phantom with details enumeration [14]: (1) high contrast bar-pattern (with variable spatial frequency in the range of $1-20 \mathrm{lp} / \mathrm{mm}$ ); (2) a series of twelve $5.6 \mathrm{~mm}$ diameter circular details with variable contrast in the range of $8.3 \%-0.15 \%$; (3) and (4) a series of eleven $0.5 \mathrm{~mm}$ diameter and eleven $0.25 \mathrm{~mm}$ diameter solid details with variable contrast in the range of $41 \%-1.4 \%$; (5) a step wedge with a ten points dynamic range scale constituted of $8 \mathrm{~mm}$ squares; (6) a micro particles step wedge (fifteen total squares with different details). 
blocks (about total $35 \mathrm{~mm}$ ) with a details plate (about $10 \mathrm{~mm}$ thick) that form together a $45 \mathrm{~mm}$ total thickness.

The details plate includes different objects [14]: a simple step wedge with variable contrast levels, one high contrast bar pattern for spatial resolution, a micro particles step wedge, a low contrast bar pattern, a series of $5.6 \mathrm{~mm}$ diameter circular details, a series of $0.5 \mathrm{~mm}$ diameter solid details and a series of $0.25 \mathrm{~mm}$ diameter solid details. Through the use of AUTOPIA software, the TORMAS phantom allows the evaluation of the following image parameters:

- integral MTF (modulation transfer function): the area under the modulation transfer function curve calculated in the high contrast bar pattern;

- MTF 50\%: the spatial resolution in $1 \mathrm{p} / \mathrm{mm}$ (line-pairs to $\mathrm{mm}$ ) correspondent to $50 \%$ level of MTF curve;

- MTF 20\%: the spatial resolution in $\mathrm{lp} / \mathrm{mm}$ (line-pairs to $\mathrm{mm}$ ) correspondent to $20 \%$ level of MTF curve;

- CNR (contrast-to-noise-ratio) of $5.6 \mathrm{~mm}$ circular details calculated as:

$$
C N R=\frac{\left|M P V_{\text {det ail }}-M P V_{\text {background }}\right|}{\sigma_{\text {background }}}
$$

where $M P V$ is mean pixel value in the detail and in the background ROI and $\sigma$ is the standard deviation in the background region;

DCC (details counts contrast) of solid circular 0.5 $\mathrm{mm}$ and $0.25 \mathrm{~mm}$ details:

$$
D C C=\frac{\sum_{i} P C N R_{i}}{n}
$$

where, according to the image of Fig. $2, P C N R_{i}$ is the contrast-to-noise-ratio of the small detail by considering the total near light points " $i$ ", represented in green color in the Fig. 2, and $n$ is the total number of the near light points;

RC (relative contrast, named simply "contrast" in the results session) of step wedge number 3 and 8 :

$$
R C=\frac{\left|M P V_{\text {det ail }}-M P V_{\text {background }}\right|}{M P V_{\text {background }}}
$$

micro-particles step wedge ratio (this ratio is named S2-3, M2-3, F2-3 for each of the three details columns, the number 2 and 3 represent the squares where the ratio is calculated, respect to the background in the same contrast square in the step wedge without details. The letters specify: S for "specks", $M$ for "microcalcifications", F for "fibers", that is the possible micro particles which simulates anatomical details in clinical mammograms):

$$
\text { ratio }=\frac{\sigma_{\text {det ail }}^{2}}{\sigma_{\text {background }}^{2}}
$$

The AUTOPIA software permits to monitor the image parameters introduced above through graphical trends and tables exportable to Excel spreadsheets [8].

In Ref. [1] Gennaro et al. reported that, the long term reproducibility control with TORMAS phantom and AUTOPIA tools has been validated: in particular it has been proved that relative contrast of step wedge is correlated to $\mathrm{kV}$ possible variations (with a tolerance of $1 \mathrm{kV}$ ), while MPV and CNR are correlated to mAs fluctuations.

To use this type of phantom for long term reproducibility check, it is necessary to discriminate

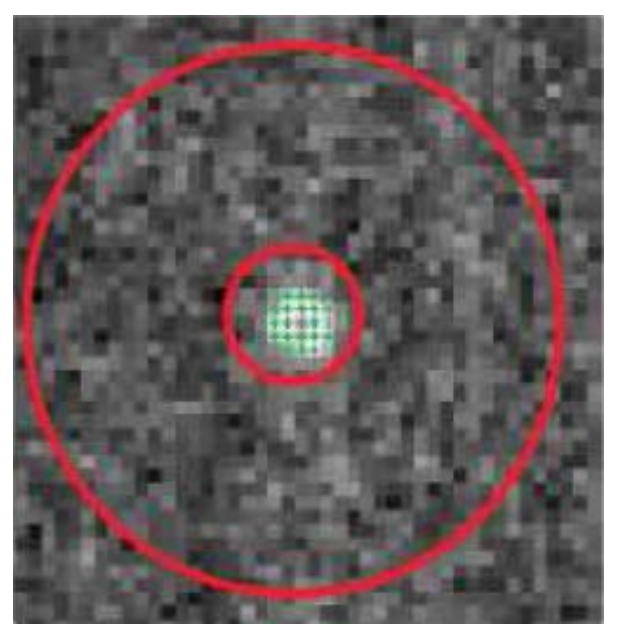

Fig. 2 Representation of the "near light points" of the small solid details in the TORMAS phantom where to calculate the contrast-to-noise-ratio and the individuation of a ROI for the measurement of background pixel-value. 
the actual results from random fluctuations: this is done through the baseline procedure performed during five successive days, with the acquisition of five images each day, at the beginning of the whole images collection. The baseline data are uploaded into AUTOPIA web page and represent the reference level for the long term reproducibility checks.

\subsection{The Mammographic Equipment}

The equipment used to acquire phantoms images is a Hologic Selenia Full Field Digital Mammography system. The size of the amorphous selenium detector is $24 \times 29 \mathrm{~cm}$ divided into $3,328 \times 4,096$ pixel: therefore the detector element size is $70 \mu \mathrm{m}$.

The X-ray tube is equipped with a tungsten anode (W) and with the two available filters for hardening the X-ray beam: $50 \mu \mathrm{m}$ rhodium $(\mathrm{Rh})$ and $50 \mu \mathrm{m}$ silver (Ag). Typically, in automatic mode for PMMA object, the $\mathrm{W} / \mathrm{Rh}$ combination is always set for common thickness, while the W/Ag is used only for thickness greater than $60 \mathrm{~mm}$ of PMMA.

\subsection{Data Collection}

Since 2011, in our center, the mammography unit described above has been monitored using weekly CDMAM phantom images (version 3.4, Artinis, St. Walburg4. 6671 AS Zetten The Netherlands) and CDMAM ver. 3.4 Analyzer software [10]. Sets of CDMAM images have been weekly carried out from January 2011 to March 2014 (for a total of 134 measurement sessions) by the same two radiographers. At the same time, the radiographers have exposed a PMMA phantom to collect SNR and mAs values. In this first phase, the automatic reading to determine the threshold contrast has been performed from a set of 6 CDMAM images as generally collected at initial stage of using the automatic reading of this phantom [11] with a $75 \%$ detection rate level.

Since Spring 2013 (second phase), in the same mammographic center a TORMAS phantom has also been used weekly because it has been provided for the long term reproducibility project by the Veneto Italian region [6]. The same two radiographers have performed setup and tests (53 total measurements with simultaneous phantom images of TORMAS, CDMAM, PMMA objects).

To test the influence of the number of CDMAM images in the reproducibility results, in a short specific period, the simultaneously acquired images for the next averaging have been increased from 6 to 20 and the detection rate has been set to $62.5 \%$, as suggested by the European Guidelines for mammographic screening, meanwhile acquiring TORMAS and PMMA phantom images too (third phase with 23 total measurements).

\subsection{Exposure Parameters}

For each phantom, the exposure conditions have been chosen by considering the type of details in the object to image and the functional properties of the digital mammographic equipment for automatic and semiautomatic mode (that is the evaluation of object thickness and density executed by the system to set the exposure parameters in automatic mode).

For the TORMAS phantom the applied exposure conditions are: semiautomatic mode by selecting $\mathrm{W} / \mathrm{Rh}$ anode/filter combination and $28 \mathrm{kV}$, compressed thickness of $45 \mathrm{~mm}$. The reference mAs product value automatically selected by the system and uploaded as baseline in AUTOPIA web page is in the range of 66-68 mAs.

For the CDMAM phantom the applied exposure conditions are: manual mode by selecting W/Rh anode/filter combination, $28 \mathrm{kV}$ and $120 \mathrm{mAs}$.

For the PMMA object the exposure conditions are those suggested by the mammographic equipment producer: semiautomatic mode by selecting $\mathrm{W} / \mathrm{Rh}$ anode/filter combination and $28 \mathrm{kV}$. The reference mAs product value automatically selected by the system is in the range of 72-76 mAs.

All possible correlations among the image parameters, obtained by the different digital images of 
phantoms considered in this work, have been evaluated and some considerations could be drawn.

\section{Results}

3.1 CDMAM Long-Term Reproducibility (First Phase Analysis)

In the first phase 134 measurements sessions have been analyzed, with the acquisition of 6 CDMAM images by setting a $75 \%$ detection rate level. In Table 1 the results of this first phase are presented: for each phantom diameter, the resulting mean contrast threshold, standard deviation SD and coefficient of variation COV have been reported. The CDMAM diameters with the SD (standard deviation) null have been excluded and the curve fitting suggests to use only diameters between $0.1 \mathrm{~mm}$ to $1 \mathrm{~mm}$ [12].

In the last rows of Table 1, the results in terms of mean gray value, standard deviation and signal-to-noise-ratio emerging from the PMMA phantom images have been reported.

The coefficient of variation of SNR and mAs parameters is lower than COV\% of CDMAM diameters. Among CDMAM parameters, only IQ seems more repeatable. Therefore, the significant correlation analyses are obtained between the parameters IQ, mAs and SNR which have lower variability than others. In this analysis the difference in exposure mode between PMMA and CDMAM is important.

The correlation can be studied between the parameters emerging from the image quality indexes and not between exposure parameters like mAs values. The correlations results have been reported in Table 2.

Despite of $\mathrm{COV} \%$ large values, a slightly negative trend of image quality parameters (contrast@0.1mm and IQ) has been showed.

In the first period of phantoms images analysis, the equipment manufacturer has performed the detector calibration twice a year. In May 2011 the software of the mammographic unit has been updated at level of pre-processing images to improve the successive image processing, in particular for prostheses. In Table 3, by considering two groups of tests performed with CDMAM and PMMA phantoms, the results show in detail the differences between before and after this update. For CDMAM phantom, 6 images have been analyzed with $75 \%$ detection rate.

Table 1 Results of CDMAM long term reproducibility during the first phase of the study.

\begin{tabular}{llll}
\hline & \multicolumn{2}{c}{ Results of the first 134 measurement sessions (CDMAM and PMMA phantoms) } \\
\cline { 2 - 4 } & Average & SD & COV\% \\
\hline IQ & 104.98 & 5.38 & 5.12 \\
CDMAM diameter & Contrast threshold $(\mu \mathrm{m})$ & & \\
$0.10 \mathrm{~mm}$ & 0.88 & 0.12 & 13.90 \\
$0.13 \mathrm{~mm}$ & 0.52 & 0.08 & 15.77 \\
$0.16 \mathrm{~mm}$ & 0.34 & 0.06 & 18.35 \\
$0.20 \mathrm{~mm}$ & 0.23 & 0.03 & 13.92 \\
$0.25 \mathrm{~mm}$ & 0.17 & 0.02 & 13.11 \\
$0.31 \mathrm{~mm}$ & 0.12 & 0.02 & 13.64 \\
$0.40 \mathrm{~mm}$ & 0.09 & 0.01 & 13.77 \\
$0.50 \mathrm{~mm}$ & 0.06 & 0.01 & 15.91 \\
$0.63 \mathrm{~mm}$ & 0.05 & 0.01 & 18.42 \\
$0.80 \mathrm{~mm}$ & 0.04 & 0.01 & 15.78 \\
$1.00 \mathrm{~mm}$ & 0.03 & 0.01 & 16.52 \\
Mean GL (Mean Gray Level PMMA) & 512.03 & 11.49 & 2.24 \\
SD PMMA & 8.59 & 0.28 & 3.24 \\
SNR PMMA & 59.69 & 2.13 & 3.56 \\
mAs PMMA & 75.01 & 2.76 & 3.69 \\
\hline
\end{tabular}


Table 2 Results of the first phase correlation analysis between CDMAM and PMMA image parameters.

\begin{tabular}{llll}
\hline First parameter & & Second parameter & R: correlation coefficient \\
\hline CDMAM IQ & vs. & SNR 40 mm PMMA & 0.75 \\
mAs 40 mm PMMA & vs. & SNR 40 mm PMMA & 0.89 \\
\hline
\end{tabular}

Table 3 Correlation analysis between CDMAM data and SNR PMMA phantom parameters before and after software upgrade.

\begin{tabular}{llll}
\hline & & \multicolumn{2}{c}{ T test before and after } \\
update software \\
\hline IQ & Mean (Group before) & Mean (Group after) & $p$-value \\
CDMAM diameter & 109.37 & 105.34 & $\mathbf{0 . 0 1 7}$ \\
$0.10 \mathrm{~mm}$ & Mean threshold contrast & Mean threshold contrast & \\
$0.13 \mathrm{~mm}$ & 0.87 & 0.90 & 0.454 \\
$0.16 \mathrm{~mm}$ & 0.46 & 0.51 & 0.170 \\
$0.20 \mathrm{~mm}$ & 0.33 & 0.31 & 0.418 \\
$0.25 \mathrm{~mm}$ & 0.22 & 0.24 & 0.178 \\
$0.31 \mathrm{~mm}$ & 0.17 & 0.16 & 0.729 \\
$0.40 \mathrm{~mm}$ & 0.12 & 0.12 & 0.583 \\
$0.50 \mathrm{~mm}$ & 0.09 & 0.09 & 0.555 \\
$0.63 \mathrm{~mm}$ & 0.06 & 0.06 & 0.601 \\
$0.80 \mathrm{~mm}$ & 0.04 & 0.05 & $\mathbf{0 . 0 2 6}$ \\
$1.00 \mathrm{~mm}$ & 0.04 & 0.04 & 0.416 \\
& 0.03 & 0.04 & 0.015 \\
Mean GL PMMA & & & $\mathbf{0 . 0 0 0}$ \\
SD PMMA & 499.72 & 519.88 & $\mathbf{0 . 0 0 0}$ \\
SNR PMMA & 9.34 & 8.57 & $\mathbf{0 . 0 0 0}$ \\
mAs PMMA & 53.50 & 60.69 & $\mathbf{0 . 0 0 0}$ \\
\hline
\end{tabular}

The statistical analysis has been performed with 2-tails T Student hypothesis test with confidence level of $95 \%$.

The bold character indicates $p$-value inferior to $5 \%$.

The statistical analysis shows that the image quality, as defined by contrast-detail CDMAM tests for almost all diameters dimensions, is the same (except for IQ data) but the other parameters, relative to PMMA phantom images, show instead statistically different values. This observation also emerges from the periodic trend reported in Fig. 3: the SNR parameter measured on the digital images of PMMA phantom significantly improves after May 2011, while the coefficients relative to CDMAM object do not show a particular trend.

\subsection{Comparison between CDMAM and TORMAS Long-Term Reproducibility (Second Phase)}

Since spring 2013, the same mammographic center has begun to use TORMAS phantom to evaluate long term reproducibility as suggested by Veneto Region Screening program [6].

The same two radiographers have acquired weekly and at the same day the PMMA, CDMAM and TORMAS phantoms digital images, so it is possible to compare the long-term reproducibility results of these three image quality control devices (53 acquisitions). This analysis is presented in Table 4: the COV\% values for CDMAM are the biggest probably because of the low number of simultaneous images acquired (6 exposures). This observation has suggested to increase the number of CDMAM images to find more reproducibility (third phase of the study). The ratio between SD and average values increases with the CDMAM diameter because the thickness of the disk diminishes and the object is not "precisely detected" from the background: the cause might be the fitting to a 


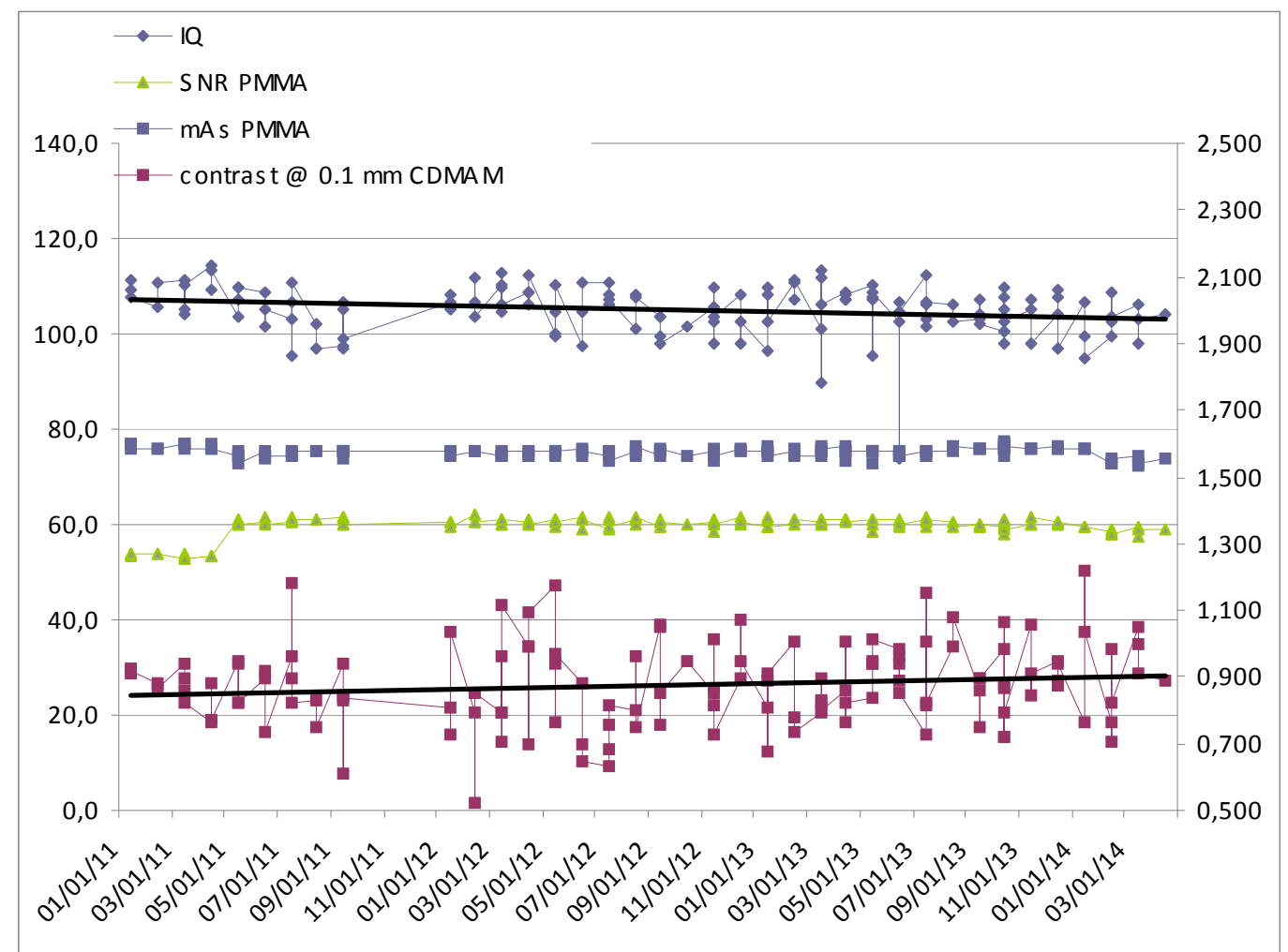

Fig. 3 Periodic trend of image quality parameters of CDMAM and PMMA phantoms. In the column at left the unit scale of SNR, mAs and IQ is present, while in the column at right the unit scale of contrast@0.1mm for CDMAM phantom is present.

Table 4 Evaluation of parameters emerging from the analyzed digital images of CDMAM, PMMA and TORMAS phantoms.

\begin{tabular}{llllllll}
\hline & \multicolumn{5}{c}{ Results of the 53 sessions (CDMAM, PMMA and TORMAS phantoms) } \\
\cline { 2 - 7 } CDMAM parameters & Mean & SD & COV\% & TORMAS parameters & Mean & SD & COV\% \\
\hline \multirow{2}{*}{ IQ } & & & & MTF 50 & 5.47 & 0.10 & 1.8 \\
& 103.67 & 6.13 & 5.9 & MTF 20 & 9.53 & 0.51 & 5.3 \\
& & & & CNR 1 & 2.98 & 0.05 & 1.8 \\
CDMAM diameter & Contrast threshold & & & CNR 2 & 1.98 & 0.04 & 2.0 \\
& & & CNR 3 & 1.48 & 0.03 & 2.0 \\
$0.10 \mathrm{~mm}$ & 0.90 & 0.11 & 12.4 & DCC 0.5-1 & 12.41 & 0.38 & 3.0 \\
$0.13 \mathrm{~mm}$ & 0.53 & 0.09 & 17.0 & DCC 0.5-2 & 8.63 & 0.28 & 3.3 \\
$0.16 \mathrm{~mm}$ & 0.35 & 0.08 & 22.3 & DCC 0.5-3 & 6.44 & 0.23 & 3.5 \\
$0.20 \mathrm{~mm}$ & 0.23 & 0.03 & 15.2 & DCC 0.2-1 & 9.32 & 0.49 & 5.2 \\
$0.25 \mathrm{~mm}$ & 0.17 & 0.03 & 15.9 & DCC 0.2-2 & 7.76 & 0.47 & 6.1 \\
$0.31 \mathrm{~mm}$ & 0.13 & 0.02 & 14.6 & DCC 0.2-3 & 5.66 & 0.47 & 8.3 \\
$0.40 \mathrm{~mm}$ & 0.09 & 0.01 & 16.5 & Contrast 3 & 63.79 & 0.25 & 0.4 \\
$0.50 \mathrm{~mm}$ & 0.06 & 0.01 & 18.6 & Contrast 8 & 27.01 & 0.13 & 0.5 \\
$0.63 \mathrm{~mm}$ & 0.05 & 0.01 & 23.1 & S 2-3 & 1.46 & 0.04 & 2.6 \\
$0.80 \mathrm{~mm}$ & 0.04 & 0.01 & 17.6 & M 2-3 & 2.40 & 0.07 & 3.0 \\
$1.00 \mathrm{~mm}$ & 0.04 & 0.01 & 18.7 & F 2-3 & 4.66 & 0.13 & 2.7 \\
PMMA parameters & Mean & SD & COV\% & PMMA parameters & Mean & SD \\
Mean GL & 506.71 & 13.59 & 2.7 & SNR & 60.01 & 0.96 & 1.6 \\
SD & 8.44 & 0.16 & 2.0 & mAs & 75.27 & 1.20 & 1.6 \\
\hline
\end{tabular}




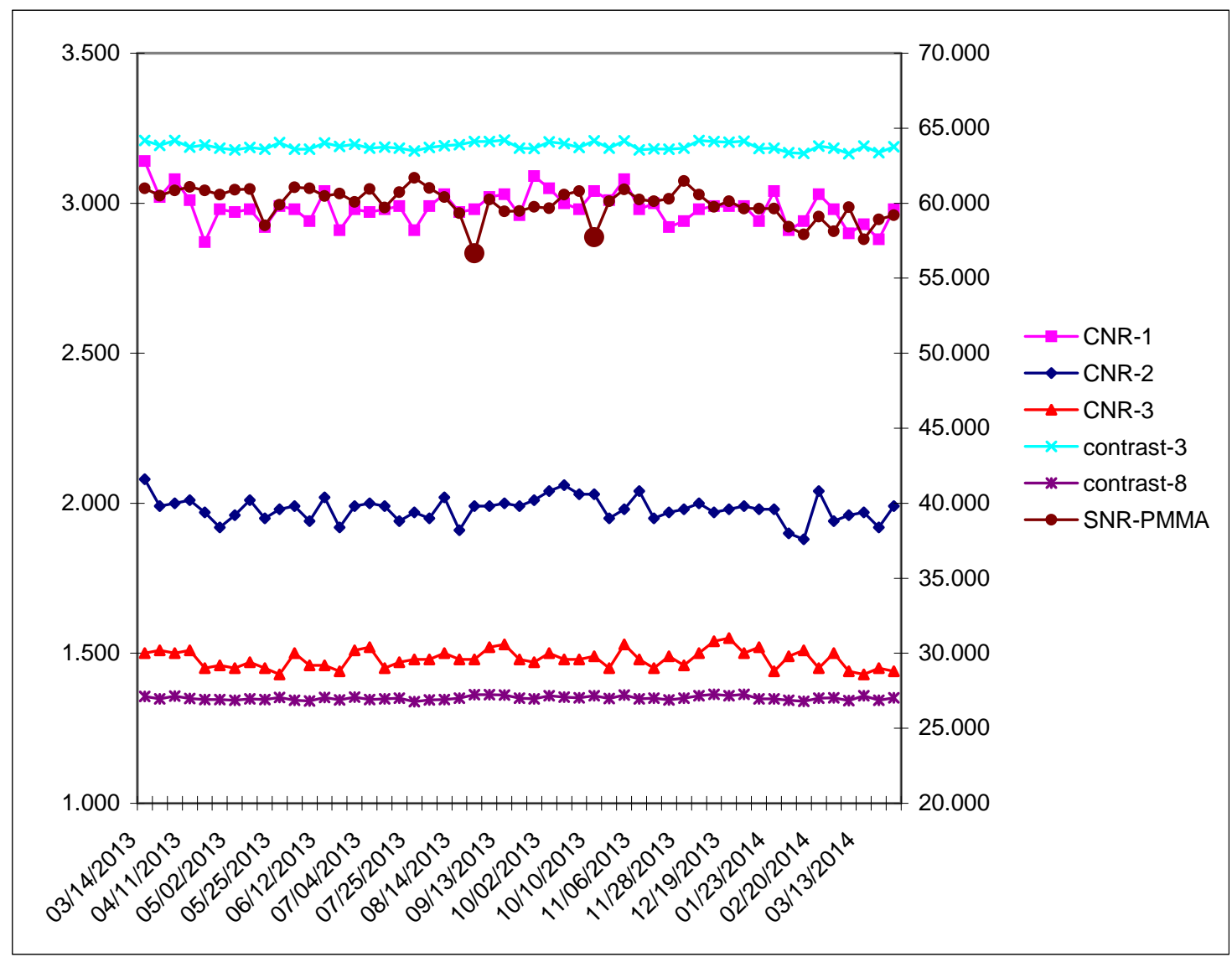

Fig. 4 Periodic trend of some TORMAS parameters with SNR measured on PMMA images: in the column at left there are reference values for CNR measured on the TORMAS digital images while in the column at right there are reference values for SNR measured on the PMMA digital images and contrast parameters calculated in the TORMAS digital images.

psychometric curve in the CDMAM procedure, which is more precise for some diameters objects than others.

Fig. 4 shows a trend of some TORMAS parameters that have the lower COV\% in Table 4.

It is necessary to underline that in some sessions (like 8.28.2013 and 10.2.2013) substantial technical problems (detector failure and compression paddle break) have occurred and have not been detected by the software AUTOPIA which has still outputted acceptable test results. On the other hand, for the same days, the tests performed through the SNR measurement (PMMA phantom) have shown a significant decreasing value (the two inferior peaks in Fig. 4).

The correlation matrix between all CDMAM and TORMAS data shows a low agreement (Table 5): in particular, the contrast that corresponds to $0.1 \mathrm{~mm}$ CDMAM diameter, typically used in contrast threshold determination, is independent to any TORMAS parameters.

\subsection{Reproducibility Increment with a Larger Number of CDMAM Images (Third Phase)}

For a short period of time the simultaneous acquired CDMAM images have been raised from 6 to 20 (23 total measurements sessions). For the last series of measurements the detection rate put in the automatic ARTINIS analyzer has been $62.5 \%$. For this period 20 CDMAM simultaneous images have been acquired and analyzed first in 6 images groups with a 75\% detection rate level (as performed initially), and later in 20 images groups with a $62.5 \%$ detection rate level (following the suggestion of the European guidelines published later than the beginning of this study [13], which indicate the collection at least of 16 images). 
Table 5 The correlation matrix between CDMAM parameters, emerging from ARTINIS Analyzer with groups of 6 images automatically analyzed with $75 \%$ detection rate, and TORMAS data extrapolated from AUTOPIA software. The bold character indicates a weak/low correlation when it exists.

\begin{tabular}{|c|c|c|c|c|c|c|c|c|c|c|c|c|c|c|c|c|}
\hline & MTF 50 & MTF 20 & CNR 1 & CNR 2 & CNR 3 & $\begin{array}{l}\text { DDC } \\
0.5-1\end{array}$ & $\begin{array}{l}\text { DDC } \\
0.5-2\end{array}$ & $\begin{array}{l}\text { DDC } \\
0.5-3\end{array}$ & $\begin{array}{l}\mathrm{DCC} \\
0.2-1\end{array}$ & $\begin{array}{l}\text { DCC } \\
0.2-2\end{array}$ & $\begin{array}{l}\mathrm{DCC} \\
0.2-3\end{array}$ & Contrast 3 & Contrast 8 & $\mathrm{~S} 2-3$ & M2-3 & F2-3 \\
\hline IQ & 0.17 & 0.38 & -0.20 & -0.24 & 0.25 & -0.31 & 0.15 & -0.18 & 0.10 & -0.17 & -0.04 & -0.04 & -0.06 & -0.05 & 0.03 & -0.04 \\
\hline $0.10 \mathrm{~mm}$ & 0.19 & 0.14 & -0.10 & 0.25 & -0.30 & 0.10 & -0.12 & 0.08 & -0.02 & -0.22 & -0.10 & -0.21 & -0.21 & 0.08 & -0.12 & -0.09 \\
\hline $0.13 \mathrm{~mm}$ & -0.36 & -0.14 & 0.32 & 0.27 & 0.14 & 0.33 & -0.12 & 0.27 & -0.01 & 0.32 & 0.24 & 0.29 & 0.25 & -0.02 & 0.04 & 0.10 \\
\hline $0.16 \mathrm{~mm}$ & -0.32 & -0.22 & 0.37 & 0.15 & -0.10 & 0.28 & -0.01 & -0.09 & 0.06 & 0.22 & 0.00 & 0.18 & 0.19 & -0.01 & 0.21 & 0.27 \\
\hline $0.20 \mathrm{~mm}$ & -0.23 & -0.30 & 0.12 & 0.14 & -0.10 & 0.24 & 0.26 & -0.10 & -0.21 & 0.13 & 0.09 & 0.02 & -0.01 & 0.15 & 0.10 & -0.02 \\
\hline $0.25 \mathrm{~mm}$ & -0.26 & -0.40 & 0.24 & 0.16 & 0.00 & 0.29 & -0.18 & -0.09 & 0.10 & 0.29 & 0.07 & 0.27 & 0.21 & 0.01 & 0.06 & 0.09 \\
\hline $0.31 \mathrm{~mm}$ & -0.15 & -0.34 & -0.09 & 0.08 & -0.00 & 0.22 & -0.10 & -0.03 & 0.08 & 0.06 & 0.04 & -0.07 & -0.08 & -0.05 & -0.07 & -0.10 \\
\hline $0.40 \mathrm{~mm}$ & -0.31 & -0.37 & 0.21 & 0.28 & -0.10 & 0.26 & -0.27 & 0.25 & 0.01 & 0.26 & 0.11 & 0.21 & 0.13 & -0.05 & -0.04 & 0.02 \\
\hline $0.50 \mathrm{~mm}$ & 0.03 & -0.55 & 0.33 & 0.21 & -0.10 & 0.29 & -0.23 & -0.08 & 0.03 & 0.27 & 0.25 & 0.13 & 0.06 & 0.00 & 0.20 & 0.29 \\
\hline $0.63 \mathrm{~mm}$ & -0.07 & -0.40 & 0.24 & 0.28 & -0.00 & 0.34 & -0.09 & -0.04 & 0.15 & 0.33 & 0.09 & 0.18 & 0.09 & 0.21 & 0.01 & 0.05 \\
\hline $0.80 \mathrm{~mm}$ & -0.25 & -0.16 & 0.25 & 0.24 & 0.22 & 0.40 & -0.15 & 0.06 & 0.02 & 0.20 & 0.00 & 0.25 & 0.12 & -0.04 & -0.21 & 0.16 \\
\hline $1.00 \mathrm{~mm}$ & -0.02 & -0.32 & 0.10 & 0.09 & -0.10 & 0.05 & 0.14 & 0.15 & -0.12 & -0.07 & -0.12 & 0.02 & 0.04 & 0.13 & 0.18 & -0.10 \\
\hline
\end{tabular}




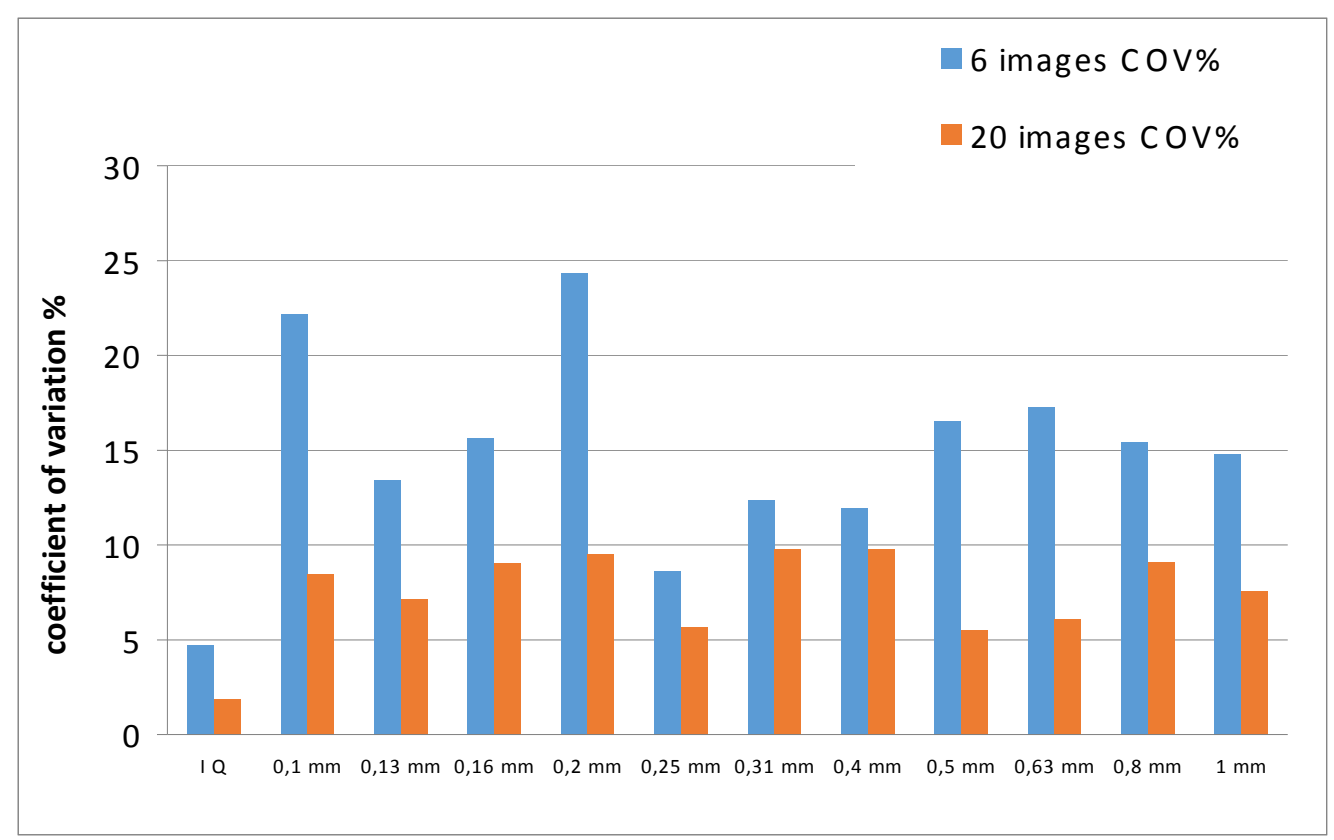

Fig. 5 COV\% about the third phase for the reproducibility of CDMAM parameters analysis. It is reported the different trend with the collection of 6 or 20 CDMAM images.

By comparing only the CDMAM images, there is an important improvement in the reproducibility parameters result deriving from the last acquisition with 20 simultaneous images and detection rate $62.5 \%$ (Fig. 5), but, despite the standard deviation decreasing, there is not any significant correlation between CDMAM data and TORMAS ones. The poor correlation result emerging from Table 5 continues to be valid also with 20 CDMAM images groups.

\section{Conclusions}

The CDMAM phantom is a useful tool to test the correct performance of a mammographic equipment or to compare different image acquisition systems in terms of contrast-detail analysis. However it seems not to be so adequate for long term reproducibility check.

The results show that working with the gold thickness correspondent to $0.1 \mathrm{~mm}$ diameter seems to be a fairly good parameter but only for the second group of 20 CDMAM images with a detection rate of $62.5 \%$. Moreover, the contrast value of $0.1 \mathrm{~mm}$ is correlated to the CDMAM image quality factor IQ, but both parametric results are independent from any
TORMAS values.

About the use of TORMAS phantom, it has been proven by other works that some parameters like CNR and DCC are well linked to mAs values, but in this case the object does not seem to perceive small mAs variation. During the implementation of the regional acquisition protocol, the AUTOPIA software has shown positive feedbacks in spite of some variations in exposure parameters. The use of TORMAS object for long term reproducibility tests leads to image quality parameters that show a substantial correlation. They do not seem very sensitive to small changes in the exposure parameters of the equipment. More statistical data are required to draw more precise conclusions.

Our long term analysis demonstrates that the simplest phantom (40 mm PMMA), through the analysis of its digital images and the SNR calculation, shows more information about mammographic system stability than the other phantoms considered (TORMAS and CDMAM).

The experience derived by working and analyzing the output data obtained from the digital images of different phantoms has delineated basic significant 
statistical data that are necessary to predict possible variations in the exposure parameters.

In conclusion, with the simple PMMA phantom and basic measurements on its digital images (SNR), it is possible to monitor the acquisition system stability and to obtain the most reliable data according to the current technological development.

\section{References}

[1] Gennaro, G., Ferro, F., Contento, G., Fornasin, F., and di Maggio, C. 2007. "Automated Analysis of Phantom Images for the Evaluation of Long-Term Reproducibility in Digital Mammography." Phys Med Biol 52: (5): 1387-407.

[2] Blot, L., Davis, A., Holubinka, M., Martì, R., and Zwiggelaar, R. 2002. "Automated Quality Assurance Applied to Mammographic Imaging." J App Signal Proc 7: 736-45.

[3] Brooks, K. W., Trueblood, J. H., Kearfott, K. J., and Lawton, D. T. 1997. "Automated Analysis of the American College of Radiology Mammographic Accreditation Phantom Image." Med Phys 24 (5): 709-23.

[4] De las Heras, H., Schöfer, F., Tiller, B., Chevalier, M., Zwettler, G., and Semturs, F. 2013. "A Phantom Using Titanium and Landolt Rings for Image Quality Evaluation in Mammography." Phys Med Biol 58: L17-L30.

[5] Perry, N., Broeders, M., de Wolf, C., Törnberg, S., Holland, R., and von Karsa, L., eds., 2006. European Guidelines for Breast Cancer Screening and Diagnosis. 4th ed.
[6] Veneto Region and Venetian Oncologic Institute. 2012. Controlli di qualità Screening mammografico. 1st ed. Padova: Cleup.

[7] Leeds Test Objects User Manual. TORMAS and TORMAX. 1997.

[8] AutoPia Software User Manual. 2012. Controlli di qualità Screening mammografico digitale.

[9] Thomas, J. A., Chakrabarti, K., Kaczmarek, R., and Romanyukha, A. 2005. "Contrast-Detail Phantom Scoring Methodology." Med Phys 32 (3): 807-14.

[10] ARTINIS CDMAM Analyser. Retrieved from www.artinis.com/product/cdmam34_analyser.

[11] Verbrugge, B. 2007. "Validation Analysis Methods for Automated CDMAM Reading." Thesis presented to graduate in the Advanced Studies of Medical Radiation Physics at the Katholieke Universiteit Leuven.

[12] Young, K. C., Alsager, A., Oduko, J. M., Bosmans, H., Verbrugge, B., Geertse, T., and van Engen, R. 2008. "Evaluation of Software for Reading Images of the CDMAM Test Object to Assess Digital Mammography Systems." Proc of SPIE 6913: 69131C-1-69131C-10.

[13] van Engen, R., Bosmans, H., Dance, D., Heid, P., Lazzari, B., Marshall, N., Schopphoven, S, Rhijssen, M, and Young, K. 2013. "Digital Mammography Update: European Protocol for the Quality Control of the Physical and Technical Aspects of Mammography Screening." Retrieved from www.euref.org..

[14] Gennaro, G., Ballaminut, A., Contento, G., and Giacobbo, M. 2015. "A Multi-parametric, Automatic Method to Monitor Long-Term Reproducibility in Digital Mammography: Results from a Regional Screening Program." Poster C-1204 European Congress of Radiology (www.myESR.org). 